\title{
Continuous versus intermittent endotracheal cuff pressure control for the prevention of ventilator-associated respiratory infections in Vietnam: study protocol for a randomised controlled trial
}

Vu Quoc Dat ${ }^{1,2}$, Ronald B. Geskus ${ }^{3,4}$, Marcel Wolbers ${ }^{4}$, Huynh Thi Loan ${ }^{5}$, Lam Minh Yen ${ }^{4}$, Nguyen Thien Binh ${ }^{6}$, Le Thanh Chien ${ }^{6}$, Nguyen Thi Hoang Mai ${ }^{4}$, Nguyen Hoan Phu ${ }^{5}$, Nguyen Phu Huong Lan ${ }^{5}$, Nguyen Van Hao ${ }^{5}$, Hoang Bao Long ${ }^{1}$, Tran Phuong Thuy ${ }^{4}$, Nguyen Van Kinh ${ }^{7}$, Nguyen Vu Trung ${ }^{7}$, Vu Dinh Phu ${ }^{7}$, Nguyen Trung Cap ${ }^{7}$, Dao Tuyet Trinh ${ }^{7}$, James Campbell ${ }^{4}$, Evelyne Kestelyn ${ }^{4}$, Heiman F. L. Wertheim ${ }^{3,8}$, Duncan Wyncoll9 Guy Edward Thwaites ${ }^{3,4}$, H. Rogier van Doorn ${ }^{1,3}$, C. Louise Thwaites ${ }^{3,4}$ and Behzad Nadjm ${ }^{1,3^{*}}$ (D)

\footnotetext{
Abstract

Background: Ventilator-associated respiratory infection (VARI) comprises ventilator-associated pneumonia (VAP) and ventilator-associated tracheobronchitis (VAT). Although their diagnostic criteria vary, together these are the most common hospital-acquired infections in intensive care units (ICUs) worldwide, responsible for a large proportion of antibiotic use within ICUs. Evidence-based strategies for the prevention of VARI in resource-limited settings are lacking. Preventing the leakage of oropharyngeal secretions into the lung using continuous endotracheal cuff pressure control is a promising strategy. The aim of this study is to investigate the efficacy of automated, continuous endotracheal cuff pressure control in preventing the development of VARI and reducing antibiotic use in ICUs in Vietnam.

(Continued on next page)
}

\footnotetext{
* Correspondence: behzadnadjm@gmail.com

${ }^{1}$ Wellcome Trust Asia Programme, Oxford University Clinical Research Unit, Hanoi, Vietnam

${ }^{3}$ Nuffield Department of Clinical Medicine, Centre for Tropical Medicine and

Global Health, University of Oxford, Oxford, UK

Full list of author information is available at the end of the article
} 
(Continued from previous page)

Methods/design: This is an open-label randomised controlled multicentre trial. We will enrol 600 adult patients intubated for $\leq 24 \mathrm{~h}$ at the time of enrolment. Eligible patients will be stratified according to admission diagnosis (180 tetanus, 420 non-tetanus) and site and will be randomised in a 1:1 ratio to receive either (1) automated, continuous control of endotracheal cuff pressure or (2) intermittent measurement and control of endotracheal cuff pressure using a manual cuff pressure meter. The primary outcome is the occurrence of VARI, defined as either VAP or VAT during the ICU admission up to a maximum of 90 days after randomisation. Patients in both groups who are at risk for VARI will receive a standardised battery of investigations if their treating physician feels a new infection has occurred, the results of which will be used by an endpoint review committee, blinded to the allocated arm and independent of patient care, to determine the primary outcome. All enrolled patients will be followed for mortality and endotracheal tube cuff-related complications at 28 days and 90 days after randomisation. Other secondary outcomes include antibiotic use; days ventilated, in ICU and in hospital; inpatient mortality; costs of antibiotics in ICU; duration of ICU stay; and duration of hospital stay.

Discussion: This study will provide high-quality evidence concerning the use of continuous endotracheal cuff pressure control as a method to reduce VARI, antibiotic use and hospitalisation costs and to shorten stay.

Trial registration: ClinicalTrials.gov, NCT02966392. Registered on November 9, 2016. Protocol version: 2.0; issue date March 3, 2017.

Keywords: Intensive care unit, Ventilator-associated pneumonia, Intubation, Tracheal tube cuff pressure, Hospital-acquired infection

\section{Background}

Hospital-acquired infections (HAIs) are the most common adverse events affecting hospitalised patients; over 700,000 occur in the United States every year, impacting length of hospital stay, cost and mortality [1]. A point prevalence survey of HAI among nearly 20,000 patients from 23 European countries in 2010 showed an HAI prevalence of $7.1 \%$, with the highest prevalence being in intensive care units (ICUs) (28.1\%) [2]. Pneumonia and other lower respiratory tract infections were the most common types of HAI, accounting for $25.7 \%$ of all HAIs [2]. HAIs were also the second most frequent indication for antibiotic treatment, with $24 \%$ of all antibiotic use being for this indication. Respiratory infection accounted for $24.8 \%$ of HAI-related antibiotic use [2]. Data on HAIs for low- and middle-income countries (LMICs) are sparse, but they indicate that the problem is even greater in this setting with the highest burden in ICUs [3]. In Vietnam HAI prevalence in ICUs has been estimated to be as high as $30 \%$, with up to $80 \%$ attributed to pneumonia and lower respiratory tract infection. High rates of broad-spectrum antibiotic use have resulted in very high levels of antibiotic resistance, especially among gramnegative bacteria [4], further necessitating the use of expensive, broad empirical therapy for HAI and contributing to the problem of antibiotic resistance. Most HAIs acquired on ICUs are ventilator-associated respiratory infections (VARIs) $[1,3]$. A substantial reduction in this would provide direct benefit to patients and their families through shorter stay, reduced costs and potentially reduced mortality. Importantly, it would also reduce antibiotic use, a key driver of antimicrobial resistance.
Critical in the pathogenesis of lung infections in intubated patients is the movement of contaminated oropharyngeal secretions into the lungs $[5,6]$. This may occur through passage internally, down the lumen of the tube, or externally around the outside of the tube, bypassing the endotracheal tube cuff. To address this, several strategies have been trialled, such as antibiotic decontamination of the oropharynx [7] (to address internal and external routes) and intermittent or continuous suctioning of secretions (subglottic suctioning, to address external routes) [8]. However, the use of antibiotic decontamination of the oropharynx, often with colistin, may cause problems in settings where infection control is limited and antibiotic resistance has reached the point that colistin is the only remaining option for many infections, or where colistin resistance among gram-negative bacteria has already emerged or been selected for. In such settings the generation and further spread of colistin-resistant gram-negative bacteria would be catastrophic, both locally and internationally. Additionally, both oropharyngeal decontamination and intermittent suctioning are labour-intensive and difficult to implement in settings where nurse-to-patient ratios are low.

Temporary falls in endotracheal cuff pressure may be a critical factor for the entry of upper airway secretions into the distal airways and lungs [6] and under-inflation of the endotracheal cuff has been associated with increased incidence of ventilator-associated pneumonia (VAP) in observational studies, with one study showing an OR of 4.3 for VAP in those patients with cuff pressure consistently $<20 \mathrm{mmHg}$ [9]. Continuous pressure 
control (CPC) minimises the periods of low cuff pressure, preventing leakage of accumulated secretions that have pooled above the cuff, whilst also preventing high pressure that can cause tracheal injury. CPC is performed with devices that are widely used in the United States and Europe, with several devices available in Vietnam, although data supporting their use as a means of reducing VARI or VAP are relatively sparse. These devices use one of two means to maintain endotracheal cuff pressure at a given level: (1) several commercially available devices use an electric pump and electronic pressure sensor to monitor and adjust cuff pressure on a moment-to-moment basis, and (2) other more recently available devices use a pneumatic system to maintain cuff pressure by applying a set pressure through the pilot balloon and cuff inflation tubing. At the time of this writing there have been three published studies that have explored the role of CPC with conventional polyvinylchloride cuffs to reduce VAP. On one hand, a small randomised controlled trial in Spain showed that whilst CPC using a modified aquarium pump did prevent falls in cuff pressure, this was associated with a small but non-significant (from $29 \%$ to $22 \%, p=0.44$ ) lowering of the incidence of clinical VAP [10]. On the other hand, a quasi-randomised prospective observational study showed a reduction in VAP from $22 \%$ to $11.2 \%(p=0.02)$ associated with $\mathrm{CPC}$ using a commercially available electronic cuff pressure controller [11]. Also, a small randomised controlled trial in a single centre in France showed a substantial and significant reduction in the occurrence of VAP during ICU stay from $26.2 \%$ to $9.8 \%(p=0.032)$ associated with a pneumatic cuff pressure controller providing CPC [12]. The designs of these studies were not optimal; all had small sample sizes, one used a very rudimentary device design, only one looked at impact on antibiotic use overall and none had blinded endpoint assessment despite a clear risk of bias with unblinded endpoints using subjective criteria. Despite these limitations, CPC appears to show the most promise as a simple intervention with minimal impact on routine nursing practice to reduce VARI, though higher-quality data are required to demonstrate its impact conclusively.

To achieve a good understanding of the value of this intervention and its cost-effectiveness, patients in the present trial will be randomised to receive either automated continuous or manual control of cuff pressure three times per day (current standard practice). This trial will produce high-quality data to inform the future care of ventilated patients in Vietnam and throughout the world.

\section{Methods/design}

Aims of the study

The aim of this trial is to determine if CPC can reduce the incidence of VARI in adults. Secondary aims include establishing whether CPC leads to an increase in proportion of ICU days not on antibiotics or a reduction in antibiotic costs, a reduction in the frequency of HAI as a whole during ICU stay, length of ventilation, length or cost of ICU and hospital stays, as well as any local complications of the endotracheal cuff and mortality at 28 and 90 days post-randomisation and at discharge from ICU and hospital.

\section{Study design}

The proposed study is an open-label, randomised controlled trial comparing tracheal cuff $\mathrm{CPC}$ via an automated electronic device with intermittent manual pressure control for the prevention of VARI in ICU. For the purpose of this study a stand-alone CPC device (reference 701; TRACOE medical GmbH, Nieder-Olm, Germany) will be used for the intervention.

\section{Study population}

Patients are being recruited over an 18-month period from the ICUs in the Hospital for Tropical Diseases (HTD) and Trung Vuong Emergency Hospital (TVH) in Ho Chi Minh City and the National Hospital of Tropical Diseases (NHTD) in Hanoi. HTD and NHTD are referral centres for the management of patients with infectious diseases. Both receive patients with infectious diseases directly from their local populations as well as patients transferred from other hospitals in southern and northern Vietnam. TVH is a provincial hospital serving the general medical needs of the local population in Ho Chi Minh City.

Patients are considered eligible for inclusion in the study when they are at least 18 years of age, have been intubated for $\leq 24 \mathrm{~h}$ at the time of randomisation (either oral or tracheostomal) and for active treatment (i.e., physician caring for patient would prescribe an antibiotic if the patient developed an infection). Exclusion criteria are previous enrolment in the study; having been previously intubated within the last 14 days; and known tracheal stenosis, tracheomalacia or stridor that is suspected secondary to physical tracheal injury. Patients are enrolled only following written informed consent provided by themselves or their legal representatives.

Patients are stratified by clinical diagnosis as tetanus or non-tetanus, because patients with tetanus represent a distinct subgroup; usually they are without significant premorbid disease or systemic inflammatory response, undergo primary tracheostomy and have a prolonged duration of intubation and ICU stay. Whilst rare in high-income settings, in many countries patients with tetanus represent a significant burden of disease with high rates of HAI and high consumption of ICU resources $[13,14]$. Thus, a protocol that attempts to address the problem of HAI in this setting also needs to specifically address tetanus. 


\section{Study endpoints}

The primary endpoint is the occurrence of VARI which is defined as VAP or ventilator-associated tracheobronchitis (VAT) during ICU stay up to a maximum of 90 days post-randomisation as evaluated by an independent endpoint committee. For both VAP and VAT, it is a core requirement for the patient to have been intubated for at least $48 \mathrm{~h}$ and for the tube to have been in place within the $48 \mathrm{~h}$ before the infection occurred. An additional core criterion for all cases is that a decision has been made to start new antibiotics or change the antibiotic regimen to treat a new infection. In addition to these core criteria, the endpoint diagnosis of VAT further requires new onset of purulent respiratory secretions or change in character of sputum or increase in volume of sputum plus either (1) temperature $>38{ }^{\circ} \mathrm{C}$ or $<36{ }^{\circ} \mathrm{C}$ or (2) white blood cell count $<4.0 \times 10^{9} / \mathrm{L}$ or $\geq 12 \times 10^{9} / \mathrm{L}$ with no other recognised cause. The endpoint diagnosis of VAP is met when the core criteria are met and there are new or progressive changes visualised by chest radiography plus two of following three criteria: (1) temperature $>38{ }^{\circ} \mathrm{C}$ or $<36{ }^{\circ} \mathrm{C}$, (2) white blood cell count $<4.0 \times 10^{9} / \mathrm{L}$ or $\geq 12 \times 10^{9} / \mathrm{L}$ with no other recognised cause, and (3) a new onset of purulent respiratory secretions or change in character of sputum or increase in volume of sputum.

Secondary endpoints include microbiologically confirmed VARI (defined as VARI plus bacterial growth of $\geq 10^{5}$ colony-forming units $/ \mathrm{ml}$ [endotracheal aspirate] or equivalent semi-quantitative growth), clinical and microbiologically confirmed VAP, proportion of intubated days receiving antibiotics, incidence of other HAI (as defined by the European Centre for Disease Prevention and Control [ECDC] criteria [15]) whilst intubated, total number of days ventilated/in ICU, cost of ICU/hospital stay, cost of antibiotics during ICU/hospital stay and mortality at 28 and 90 days after randomisation as well as at ICU and hospital discharge. Safety data, including in-hospital re-intubation, tracheomalacia, tracheal stenosis and other local complications of endotracheal placement, will be collected up to 90 days post-enrolment or until hospital discharge, whichever is later. The time periods over which endpoints or censoring events will be elicited are shown in Table 1.

\section{Sample size}

Based on local data and data derived from a large point prevalence study in Vietnam [4], the estimated prevalence of VARI (the primary outcome) in non-tetanus and tetanus ventilated patients is $20 \%$ and $30 \%$, respectively. We expect an effect size of a $50 \%$ reduction in VARI using CPC, but we consider that a $40 \%$ reduction would be the minimum we would wish to detect $[11,12]$. This effect size is expected to be the same in both tetanus and non-tetanus patients. To preserve the generalisability of the study for settings where tetanus is less common whilst still demonstrating the utility of the intervention in this patient group, we will stratify randomisation to ensure that $30 \%$ of our recruited patients will have a diagnosis of tetanus. Thus we might expect to reduce VARI rates from $20 \%$ to $12 \%$ in non-tetanus patients and from $30 \%$ to $18 \%$ in tetanus patients. In a study population with $30 \%$ tetanus patients, this corresponds to an absolute

Table 1 Follow-up periods for all study endpoints

\begin{tabular}{|c|c|c|}
\hline Endpoint & $\begin{array}{l}\text { Beginning of } \\
\text { follow-up }\end{array}$ & $\begin{array}{l}\text { End of follow-up/censoring event } \\
\text { (soonest event applies, except where specified) }\end{array}$ \\
\hline \multicolumn{3}{|l|}{ Primary } \\
\hline VARI & Randomisation & ICU discharge/death/transfer or 90 days \\
\hline \multicolumn{3}{|l|}{ Secondary } \\
\hline Microbiologically confirmed VARI & Randomisation & ICU discharge/death/transfer or 90 days \\
\hline Clinical and microbiologically confirmed VAP & Randomisation & ICU discharge/death/transfer or 90 days \\
\hline Intubated days not receiving antibiotics & Randomisation & ICU discharge/death/transfer or 90 days \\
\hline Incidence of $\mathrm{HAl}$ & Randomisation & Extubation/death/transfer/discharge from ICU or 90 days \\
\hline Days ventilated/in ICU & Randomisation & ICU discharge, death, transfer \\
\hline Cost of ICU stay & ICU admission & ICU discharge/death/transfer \\
\hline Cost of antibiotics in ICU stay & ICU admission & ICU discharge/death/transfer \\
\hline Cost of hospital stay & Hospital admission & Hospital discharge \\
\hline 28-Day mortality & Randomisation & 28 Days after randomisation \\
\hline 90-Day mortality & Randomisation & 90 Days after randomisation \\
\hline ICU mortality & Randomisation & Discharge from ICU or death/palliative discharge from ICU \\
\hline Hospital mortality & Randomisation & Discharge from hospital or death/palliative discharge from hospital \\
\hline
\end{tabular}


risk reduction from $23 \%$ to $13.8 \%$. To detect this reduction with $80 \%$ power at the two-sided 5\% significance level, 278 patients are required in each arm. Allowing for $8 \%$ loss to follow-up or early extubation (before $48 \mathrm{~h}$ ), we aim to recruit 600 patients in total (420 non-tetanus, 180 tetanus). To our knowledge, this will make it the largest single trial addressing the prevention of VARI through CPC.

\section{Randomisation procedure}

Randomisation will be stratified 1:1 by site and whether the patient has a clinical diagnosis of tetanus at the time of randomisation. A stratified, computer-generated randomisation list will be created using block randomisation with variable block length and incorporated into secure internet-accessible software that implements the randomisation. Once an eligible patient has consented, the initials and date of birth of the patient will be entered into the software by study staff. On the basis of the randomisation list, the software will produce the treatment allocation, which will be displayed and recorded in the study database. All entries and outputs of the software will be auditable.

\section{Intervention}

Depending on the results of randomisation, patients will receive either manual, intermittent endotracheal cuff pressure, which is checked and adjusted 8-hourly (standard care), or automatic, continuous endotracheal CPC (intervention group). Cuff pressure will be recorded in every ventilated patient every $8 \mathrm{~h}$. Target pressure in both groups will be $25 \mathrm{cmH}_{2} \mathrm{O}$ as a default. Changes in this target will be recorded, and the clinical reason for the change will be noted.

\section{Study assessment}

On a daily basis, the attending physicians will assess whether there is a new infection in a patient who has been intubated for $\geq 48 \mathrm{~h}$ and in whom the tube is still in place or has been removed within the previous $48 \mathrm{~h}$. If the answer is 'yes', a standard battery of tests, including complete blood count, procalcitonin, arterial blood gas, blood culture, sputum/endotracheal aspirate microscopy and culture, urine culture and chest $x$-ray, will be performed regardless of the suspected site of infection. Test results and clinical details from up to 5 days previous, the day of the HAI evaluation and the subsequent 2 days will be collected, including maximum/minimum temperature, changes in ventilation parameters (fraction of inspired oxygen, positive end-expiratory pressure), changes in sputum colour or volume, and new inotrope or vasopressor requirements. The treating clinicians will provide their own diagnosis of the aetiology of the infection according to ECDC criteria and will prescribe antibiotic and other therapies as per routine care. However, for the primary endpoint, an endpoint review committee, blinded to the treatment allocation and independent of clinical involvement with the patient, will review the complete case report form (CRF) and radiology of patients completing the study at the end of each month to determine whether they met the primary or appropriate secondary endpoint criteria.

Enrolled patients will have cuff pressure controlled in accordance with the allocated study arm for the entire duration of their intubated time on ICU. Follow-up for the primary outcome will be until transfer to another facility, death, discharge from ICU or 90 days following randomisation, whichever is soonest. Patients who require re-intubation during the same stay in ICU will continue in the study, using the same cuff pressure control measures originally allocated to them. Patients discharged from ICU but requiring return to ICU and reintubation will not be re-enrolled and will be managed in accordance with standard care. At the end of the hospital stay the costs of the stay as billed will be recorded (including bed, ventilator and other supportive therapy costs, drugs including antibiotic costs, but not including labour as per Vietnamese Ministry of Health guidance). Additionally, as part of secondary endpoint assessment, study staff will telephone patients or their relatives at 28 and 90 days after randomisation if they are no longer inpatients in order to assess mortality and complications related to intubation. A flowchart of study procedures is shown in Fig. 1; a schedule of enrolment, interventions and assessments is provided in Fig. 2.

\section{Data management}

Relevant data will be recorded onto a CRF and checked for accuracy. After the follow-up is finished the CRFs will be de-identified by removing patient identifiers and telephone numbers. Selected staff will be trained in how to enter all clinical data from the CRFs and from laboratory source documents into a computerised data entry system using double data entry. Data will be retained for $\geq 15$ years on completion of the study (last patient followed for 90 days). Original paper documents will be maintained for $\geq 5$ years; thereafter digital copies will be retained. All stored records will be kept secure and confidential.

All personal information reviewed as part of this study will remain confidential. Patient names or identifiers will not appear in any database. Any scientific publications or reports will not include information that can identify any patient. Oxford University Clinical Research Unit (OUCRU) is committed to open access to study data and is working with its partners to facilitate mechanisms to enable this. 


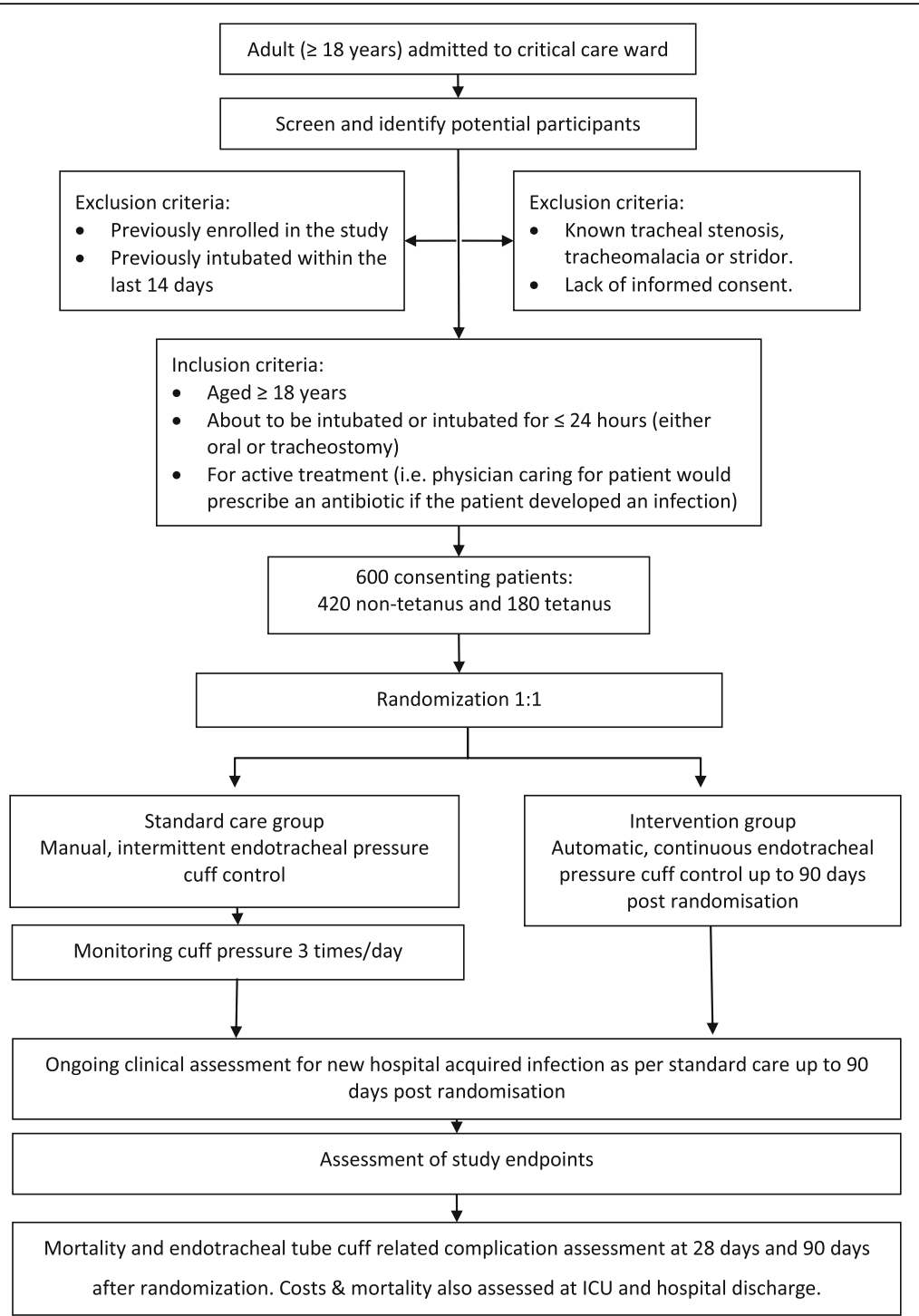

Fig. 1 Flowchart of study procedures

\section{Statistical analysis}

The details regarding all planned analyses will be elaborated in an analysis plan prior to analysis. Reporting of the trial results will clearly indicate which analyses were part of this plan and which were not. The effect of the intervention on the primary outcome will be quantified in an intention-to-treat analysis that allocates all patients according to the randomised treatment arm. The primary endpoint will additionally be analysed in a per-protocol analysis which will exclude patients not fulfilling the eligibility criteria and patients intubated for $<48 \mathrm{~h}$.

The primary endpoint will be analysed using a logistic regression model with the randomised arm as the main covariate and adjustment for tetanus status. Patients who are intubated for $<48 \mathrm{~h}$ will be regarded as not having reached the primary endpoint. Potential heterogeneity of the intervention effect will be assessed on the basis of appropriate interaction (likelihood ratio) tests and the predefined subgroups, including (1) patients with and without tetanus, (2) patients intubated for $\leq 2$ and $>2 \mathrm{~h}$ before randomisation, (3) patients with and without tracheostomy and (4) hospital site.

Binary secondary endpoints (clinical and microbiologically confirmed VAP) will be analysed in the same way as the primary endpoint. Both the distribution of duration of ventilation (i.e., time to extubation) and the time of ICU stay will be estimated. Death will be considered as a competing event; hence we will estimate the cause-specific cumulative incidence. Cause-specific cumulative incidence functions will be compared between the arms using a Fine-Gray regression model with the treatment arm as the main covariate and adjustment for 


\begin{tabular}{|c|c|c|c|c|c|c|c|c|c|}
\hline \multirow[b]{3}{*}{ TIMEPOINT** } & \multicolumn{9}{|c|}{ STUDY PERIOD } \\
\hline & \multirow[t]{2}{*}{ Enrolment } & \multirow[t]{2}{*}{ Allocation } & \multicolumn{6}{|c|}{ Post-allocation } & \multirow{2}{*}{$\begin{array}{c}\text { Close-out } \\
\\
\text { ᄋ } \\
\text { वे }\end{array}$} \\
\hline & & & 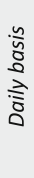 & 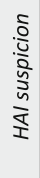 & 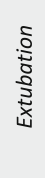 & 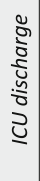 & 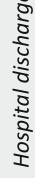 & $\begin{array}{l}\text { Nे } \\
\text { डे } \\
\text { वे }\end{array}$ & \\
\hline \multirow{4}{*}{$\begin{array}{l}\text { ENROLMENT: } \\
\text { Eligibility screen } \\
\text { Informed consent } \\
\text { Randomisation }\end{array}$} & & & & & & & & & \\
\hline & $x$ & & & & & & & & \\
\hline & $x$ & & & & & & & & \\
\hline & & $\mathrm{x}$ & & & & & & & \\
\hline \multirow{3}{*}{$\begin{array}{l}\text { INTERVENTIONS: } \\
\text { Continuous endotracheal } \\
\text { cuff pressure control } \\
\text { Intermittent endotracheal } \\
\text { cuff pressure control (g8h) }\end{array}$} & & & & & & & & & \\
\hline & & $x$ & & & & & & & \\
\hline & & $x$ & & & & & & & \\
\hline \multicolumn{10}{|l|}{ ASSESSMENTS: } \\
\hline \multirow{3}{*}{$\begin{array}{l}\text { Demographic data } \\
\text { Charlson Comorbidity Index } \\
\text { \& APACHE II } \\
\text { PEEP and FiO2 }\end{array}$} & $x$ & & & & & & & & \\
\hline & $\mathrm{x}$ & & & & & & & & \\
\hline & & $x$ & $x$ & $x$ & & & & & \\
\hline \multirow{4}{*}{$\begin{array}{l}\text { HbA1c } \\
\text { Chest x-ray } \\
\text { Cuff pressure measurement } \\
\text { Complete Blood Count }\end{array}$} & & $\mathrm{x}$ & & & & & & & \\
\hline & & $\mathrm{x}$ & & $\mathrm{x}$ & & & & & \\
\hline & & $x$ & $x$ & & & & & & \\
\hline & & $\mathrm{x}$ & & $\mathrm{x}$ & & & & & \\
\hline \multirow{4}{*}{$\begin{array}{l}\text { Arterial blood gas analysis } \\
\text { Procalcitonin } \\
\text { Blood and urine culture } \\
\text { Respiratory secretions } \\
\text { microscopy \& culture }\end{array}$} & & $\mathrm{x}$ & & $\mathrm{x}$ & & & & & \\
\hline & & & & $\mathrm{x}$ & & & & & \\
\hline & & & & $\mathrm{x}$ & & & & & \\
\hline & & & & $\mathrm{x}$ & & & & & \\
\hline \multicolumn{10}{|l|}{$\begin{array}{r}\text { Risk factors for VARI } \\
\text { Use of sedative agents } \\
\text { Use of paralytic agents } \\
\text { Feeding } \\
\text { Use of PPI }\end{array}$} \\
\hline \multirow{4}{*}{$\begin{array}{l}\text { Mortality } \\
\text { Endotracheal tube cuff } \\
\text { related complications } \\
\text { Cost of stay } \\
\text { Cost of antibiotics }\end{array}$} & & & $x$ & & & $x$ & $x$ & $x$ & $x$ \\
\hline & & & $x$ & & & $x$ & $x$ & $x$ & $x$ \\
\hline & & & & & & $x$ & $x$ & & \\
\hline & & & & & & $x$ & $x$ & & \\
\hline
\end{tabular}

Fig. 2 Schedule of enrolment, interventions and assessments. HAl Hospital-acquired infection, ICU Intensive care unit, APACHE Acute Physiology and Chronic Health Evaluation, PEEP Positive end-expiratory pressure, FiO ${ }_{2}$ Fraction of inspired oxygen, HbAlc Glycated haemoglobin,

VARI Ventilator-associated respiratory infection, PPI Proton pump inhibitor

tetanus status. Mortality will be visualised in each arm (overall and by tetanus status) using Kaplan-Meier curves and modelled using Cox regression. The proportion of intubated days free of antibiotics will be analysed using a Poisson regression model with the number of intubated days without antibiotics as the outcome, the randomised arm as the main covariate, and the (log-transformed) total number of intubated days as an offset. Quasi-likelihood will be used to account for potential over-dispersion.

The frequency of adverse events will be summarised (in terms of both the total number of events and the number of patients with at least one event). The proportion of patients with at least one adverse event (overall and for each specific event separately) will be summarised and (informally) compared between the two treatment groups using Fisher's exact test.

\section{Reporting of adverse events and monitoring}

Because adverse events are relatively common in critically ill patients intubated in the ICU, safety reporting will focus on events of potential relevance to cuff pressure control. The events being reported to the ethics committees are (1) all unexpected serious adverse events, (2) all serious adverse events judged to be related or possibly related to cuff pressure and (3) all deaths or palliative discharges (discharge to home of a patient where ongoing care was considered futile to permit the patient to die at home, a commonly preferred alternative to dying in hospital in Vietnam). Reporting to the ethics committees will be done in accordance with good clinical practice.

An independent data and safety monitoring board (DSMB) has been established, consisting of expert 
Vietnamese and international researchers and doctors with the necessary clinical, research and statistical knowledge. The DSMB has reviewed the protocol, and a DSMB charter outlines its responsibilities and how it operates. All DSMB reports will be sent to the responsible ethics committees, including the site ethics committees and the Oxford Tropical Research Ethics Committee, for consideration. Recruitment will continue at active sites during the DSMB review period. No interim analyses are planned for the primary endpoint; however, safety analyses will be conducted after 30 patients have been recruited and subsequently at points decided by the DSMB.

The study will be monitored by a team at OUCRU, independent of study design and management. Central monitoring will take place regularly. On-site monitoring occurred after the recruitment of 25 patients in total, with a second monitoring visit planned after recruitment of 200 patients, and further monitoring will be scheduled depending on the results of early monitoring visits. The SPIRIT (Standard Protocol Items: Recommendations for Interventional Trials) for this research is available in Additional file 1.

\section{Discussion}

To our knowledge, this is the first study with an adequate sample size and robustly designed endpoints to evaluate the effectiveness of endotracheal cuff CPC in preventing VARI. It also concurrently measures the impact of the intervention on important patient-centred clinical outcomes and important outcomes concerning antibiotic use. An ideal intervention should, in addition to an impact on the primary outcome (VARI episodes), show resultant benefits in reducing mortality, duration of mechanical ventilation and ICU stay in association with reducing antibiotic use, as recently recommended for antibiotic stewardship programmes [16]. The intervention will likely be considered of questionable benefit should it result only in a reduction in VARI episodes without corresponding reductions in at least some of these patient-centred or antibiotic stewardship outcomes.

Leakage of secretions through the endotracheal tube cuff is commonplace in clinical practice with intermittent cuff pressure monitoring [17]. Approaches to prevent aspiration of contaminated oropharyngeal secretions by maintaining endotracheal cuff pressure have been studied in an effort to reduce VAP rate and its associated mortality. However, previous studies (small and non-randomised controlled trials) have not established a sufficient evidence base on which to recommend CPC routinely $[18,19]$. This study provides an opportunity to evaluate the impact of CPC in preventing VARI and in reducing antibiotic use as an aspect of antimicrobial stewardship in a resource-constrained setting with established high levels of antibiotic use and resistance.

Pathogen colonisation of the trachea and endotracheal tube may progress to VAT, which can be a transitional stage before development of VAP. Both VAT and VAP share common clinical manifestations of fever, purulent respiratory secretions, and leucocytosis with the distinguishing presence of pulmonary infiltrates on chest $x$ rays in VAP. In a prospective study in 10 countries with 114 ICUs, incidence of VAT was similar to VAP $(11 \%$ vs 12\%) [20]. However, VAT is often a predisposing factor for development of VAP, and appropriate antibiotic treatment for VAT can reduce the progression to VAP $[20,21]$. In a survey of perceptions of international physicians, nearly $80 \%$ of respondents diagnosed VAT on the basis of both clinical and microbiological criteria, and the remaining respondents used clinical criteria alone or as a diagnosis of exclusion. Half of the respondents agreed to antibiotic use in patients with VAT [22]. In Vietnam it is common practice to treat VAT, and in view of these considerations, we constructed our trial around an endpoint that encompassed both VAT and VAP, in contrast to other studies [10-12]. Although recognition of VAT and intervention at this stage are expected to impact the mortality, length of stay and treatment cost which would be attributed to VAP, it is clear that there are important differences between surveillance definitions and actual clinical definitions on which treatment is based.

In an open-label study where the treating doctor is not blinded to the intervention, there is a risk of clinical ascertainment bias of the study endpoint [23]. Whilst death or microbiological results are hard endpoints which are unlikely to be biased by a lack of blinding, VARI diagnostic categories and antibiotic use are more subjective and vulnerable to misclassification bias in non-blinded trials [24]. For this reason an independent endpoint committee is recommended for multicentre clinical trials to judge complex, multi-aspect definitions and outcomes $[25,26]$. In our study treating doctors know the allocated arm of the patient and perform assessments for HAI as part of routine care; therefore the use of a blinded endpoint committee will reduce bias in the primary endpoint evaluation. The study may also offer an opportunity to understand inconsistencies in the assessment of VARI diagnoses between treating doctors and the endpoint committee, and within the endpoint committee, as well as how this impacts the significance of results.

A future consideration would be to evaluate the impact of a successful intervention at the ICU level, on the speed at which resistance develops in the unit, with the hope that a substantial reduction in the use of antibiotics 
would be reflected in slower development of resistance in the ICU as a whole. This would require a clusterrandomised or stepped-wedge design, and such an approach should be considered for any potential rollout of a successful intervention. Given the crisis in antibiotic resistance, this would provide valuable additional information not attainable through an individual patient randomised trial design to complement the results of this study.

\section{Trial status}

The trial is currently recruiting. Important protocol amendments will be communicated to relevant bodies (investigators, institutional review boards, DSMB, journals) by Dr. B. Nadjm, trial principal investigator, as soon as changes are made.

\section{Trial sponsor}

Oxford University - Centre for Tropical Medicine (sponsor's reference R45398/RE001). Contact: Evelyne Kestelyn, 764 Vo Van Kiet Street, Ward 1, District 5, Ho Chi Minh City, Vietnam. Telephone: + 84839237954. Email: info@oucru.org. The sponsor has specialised insurance to cover any potential harm caused as a result of participation in the study.

\section{Additional file}

Additional file 1: Standard Protocol Items: Recommendations for Interventional Trials (SPIRIT) 2013 checklist: recommended items to address in a clinical trial protocol and related documents. (DOCX $51 \mathrm{~kb}$ )

\section{Acknowledgements}

We thank the staff at HTD, TVH, NHTD and OUCRU for their support.

\section{Funding}

The study is funded by the Oxford University Clinical Research Unit in Vietnam through its Wellcome Trust Major Overseas Programme Grant (106680/Z/14/Z). The study funder has no input into any aspect of the study.

\section{Availability of data and materials}

Not applicable.

\section{Authors' contributions}

BN, VQD and DW conceived of the study and were involved in study design, the application to the research ethics committee and the drafting of the manuscript. MW and RBG provided statistical expertise for the study design and were involved in drafting the manuscript. JC, PHL, NVH, VDP, NTC, DTT, NVK, NVT, HRVD, EK, HFLW, GT and CLT were involved in study design and in reviewing the manuscript. VQD, HTL, LMY, NTB, HBL, TPT and LTC were involved in study design and the application to the local institutional review board (IRB)/ethics committee. VQD, LMY, NTHM, NHP and NTB participate in patient recruitment. JC, PHL, DTT supervise and perform microbiological analyses. BN, VQD, HRVD, LTC and LMY participate as members of the endpoint review committee. VQD, LYM, NTB, HBL, TPT, LTC and BN are members of the trial management team. All authors read and approved the final manuscript.

\section{Ethics approval and consent to participate}

Ethical approval was obtained through the Oxford Tropical Research Ethics Committee (OxTREC) (reference 26-16), the Tropical Diseases Hospital in Ho Chi Minh City (reference number 32/HDDD), Trung Vuong Emergency Hospital (reference number 670/BVTV) and National Hospital of Tropical Diseases (reference number 22/HDDD-NDTU). The original approved version of this study did not have a 90-day limit on the intervention and follow-up for endpoints; these changes have been approved in amendments. Patients who require intubation at the study site on or after arrival or who have been intubated prior to transfer, or their legal representatives, will be approached as soon as is possible in line with prioritising patient care, fully informed, and invited to consent to the study. Consent will be obtained by study or hospital staff trained in good clinical practice and the consent procedure. Patients will be enrolled only after informed consent has been obtained. Randomisation will then take place, and patients will be managed in the appropriate manner. Patient care will never be delayed for consent or randomisation procedures. Where consent is provided by a patient's representative and they later regain the capacity to provide consent, the patient will be approached and given the opportunity to withdraw from the study.

\section{Consent for publication}

Not applicable.

\section{Competing interests}

DW has served as a board member for Astellas; lectured for HealthCare 21, Astellas, Pfizer, Sage, Johnson \& Johnson, and Bioproducts; received support for the development of educational presentations from Astellas; and received funding from Pfizer (board member) and Astellas (board member) as well as Sage, Healthcare21, Johnson \& Johnson and Bioproducts speaker bureaus (all unrelated to this work). The other authors declare that they have no competing interests.

\section{Publisher's Note}

Springer Nature remains neutral with regard to jurisdictional claims in published maps and institutional affiliations.

\section{Author details}

${ }^{1}$ Wellcome Trust Asia Programme, Oxford University Clinical Research Unit, Hanoi, Vietnam. ${ }^{2}$ Department of Infectious Diseases, Hanoi Medical University, Hanoi, Vietnam. ${ }^{3}$ Nuffield Department of Clinical Medicine, Centre for Tropical Medicine and Global Health, University of Oxford, Oxford, UK. ${ }^{4}$ Wellcome Asia Programme, Oxford University Clinical Research Unit, Ho Chi Minh City, Vietnam. ${ }^{5}$ The Hospital for Tropical Diseases, Ho Chi Minh City, Vietnam. ${ }^{6}$ Trung Vuong Hospital, Ho Chi Minh City, Vietnam. ${ }^{7}$ National Hospital for Tropical Diseases, Hanoi, Vietnam. ${ }^{8}$ Department of Medical Microbiology, Radboud University Medical Centre, Nijmegen, The Netherlands. 'Department of Adult Critical Care, Guy's and St Thomas' NHS Foundation Trust, St Thomas' Hospital, London, UK

Received: 3 August 2017 Accepted: 12 March 2018

Published online: 04 April 2018

References

1. Magill SS, Edwards JR, Bamberg W, Beldavs ZG, Dumyati G, Kainer MA, Lynfield R, Maloney M, McAllister-Hollod L, Nadle J, et al. Multistate pointprevalence survey of health care-associated infections. N Engl J Med. 2014; 370(13):1198-208

2. Zarb P, Coignard B, Griskeviciene J, Muller A, Vankerckhoven V, Weist K, Goossens M, Vaerenberg S, Hopkins S, Catry B, et al. The European Centre for Disease Prevention and Control (ECDC) pilot point prevalence survey of healthcare-associated infections and antimicrobial use. Euro Surveill. 2012; 17(46):20316.

3. Allegranzi B, Bagheri Nejad S, Combescure C, Graafmans W, Attar H, Donaldson L, Pittet D. Burden of endemic health-care-associated infection in developing countries: systematic review and meta-analysis. Lancet. 2011; 377(9761):228-41.

4. Phu VD, Wertheim HF, Larsson M, Nadjm B, Dinh QD, Nilsson LE, Rydell U, Le TT, Trinh SH, Pham HM, et al. Burden of hospital acquired infections and antimicrobial use in Vietnamese adult intensive care units. PLoS One. 2016; 11(1):e0147544

5. Safdar N, Crnich CJ, Maki DG. The pathogenesis of ventilator-associated pneumonia: its relevance to developing effective strategies for prevention. Respir Care. 2005;50(6):725-39. discussion 739-41

6. Zolfaghari PS, Wyncoll DL. The tracheal tube: gateway to ventilatorassociated pneumonia. Crit Care. 2011;15(5):310. 
7. Labeau SO, Van de Vyver K, Brusselaers N, Vogelaers D, Blot SI. Prevention of ventilator-associated pneumonia with oral antiseptics: a systematic review and meta-analysis. Lancet Infect Dis. 2011;11(11):845-54.

8. Frost SA, Azeem A, Alexandrou E, Tam V, Murphy JK, Hunt L, O'Regan W, Hillman KM. Subglottic secretion drainage for preventing ventilator associated pneumonia: a meta-analysis. Aust Crit Care. 2013;26(4):180-8.

9. Rello J, Sonora R, Jubert P, Artigas A, Rue M, Valles J. Pneumonia in intubated patients: role of respiratory airway care. Am J Respir Crit Care Med. 1996;154(1):111-5.

10. Valencia M, Ferrer M, Farre R, Navajas D, Badia JR, Nicolas JM, Torres A. Automatic control of tracheal tube cuff pressure in ventilated patients in semirecumbent position: a randomized trial. Crit Care Med. 2007;35(6):1543-9.

11. Lorente L, Lecuona M, Jimenez A, Lorenzo L, Roca I, Cabrera J, Llanos C, Mora ML. Continuous endotracheal tube cuff pressure control system protects against ventilator-associated pneumonia. Crit Care. 2014;18(2):R77.

12. Nseir S, Zerimech F, Fournier C, Lubret R, Ramon P, Durocher A, Balduyck M. Continuous control of tracheal cuff pressure and microaspiration of gastric contents in critically ill patients. Am J Respir Crit Care Med. 2011;184(9):1041-7.

13. Thwaites CL, Farrar JJ. Preventing and treating tetanus. BMJ. 2003;326(7381): $117-8$.

14. Kyu HH, Mumford JE, Stanaway JD, Barber RM, Hancock JR, Vos T, Murray CJ, Naghavi M. Mortality from tetanus between 1990 and 2015: findings from the Global Burden of Disease study 2015. BMC Public Health. 2017;17(1):179.

15. European Centre for Disease Prevention and Control (ECDC). European surveillance of healthcare-associated infections in intensive care units. HAlNet ICU protocol version 1.02. 2015. http://ecdc.europa.eu/en/publications/ Publications/healthcare-associated-infections-HAI-ICU-protocol.pdf.

16. Barlam TF, Cosgrove SE, Abbo LM, MacDougall C, Schuetz AN, Septimus EJ, Srinivasan A, Dellit TH, Falck-Ytter YT, Fishman NO, et al. Implementing an antibiotic stewardship program: guidelines by the Infectious Diseases Society of America and the Society for Healthcare Epidemiology of America. Clin Infect Dis. 2016;62(10):e51-77.

17. Carter EL, Duguid A, Ercole A, Matta B, Burnstein RM, Veenith T. Strategies to prevent ventilation-associated pneumonia: the effect of cuff pressure monitoring techniques and tracheal tube type on aspiration of subglottic secretions: an in-vitro study. Eur J Anaesthesiol. 2014;31(3):166-71.

18. Tablan OC, Anderson $\perp$, Besser R, Bridges C, Hajjeh R. Guidelines for preventing health-care-associated pneumonia, 2003: recommendations of CDC and the Healthcare Infection Control Practices Advisory Committee. MMWR Recomm Rep. 2004;53(RR-3):1-36.

19. Torres A, Ewig S, Lode H, Carlet J. Defining, treating and preventing hospital acquired pneumonia: European perspective. Intensive Care Med. 2009;35(1):9-29.

20. Martin-Loeches I, Povoa P, Rodriguez A, Curcio D, Suarez D, Mira JP, Cordero ML, Lepecq R, Girault C, Candeias C, et al. Incidence and prognosis of ventilator-associated tracheobronchitis (TAVeM): a multicentre, prospective, observational study. Lancet Respir Med. 2015;3(11):859-68.

21. Nseir S, Martin-Loeches I, Makris D, Jaillette E, Karvouniaris M, Valles J, Zakynthinos E, Artigas A. Impact of appropriate antimicrobial treatment on transition from ventilator-associated tracheobronchitis to ventilatorassociated pneumonia. Crit Care. 2014;18(3):R129.

22. Rodriguez A, Povoa P, Nseir S, Salluh J, Curcio D, Martin-Loeches I. Incidence and diagnosis of ventilator-associated tracheobronchitis in the intensive care unit: an international online survey. Crit Care. 2014;18(1):R32.

23. Schulz KF, Grimes DA. Blinding in randomised trials: hiding who got what. Lancet. 2002;359(9307):696-700.

24. Dechartres A, Boutron I, Roy C, Ravaud P. Inadequate planning and reporting of adjudication committees in clinical trials: recommendation proposal. J Clin Epidemiol. 2009;62(7):695-702.

25. Naslund U, Grip L, Fischer-Hansen J, Gundersen T, Lehto S, Wallentin L. The impact of an end-point committee in a large multicentre, randomized, placebo-controlled clinical trial: results with and without the end-point committee's final decision on end-points. Eur Heart J. 1999;20(10):771-7.

26. Moher D, Schulz KF, Altman DG. The CONSORT statement: revised recommendations for improving the quality of reports of parallel-group randomised trials. Lancet. 2001:357(9263):1191-4.

\section{Submit your next manuscript to BioMed Central and we will help you at every step:}

- We accept pre-submission inquiries

- Our selector tool helps you to find the most relevant journal

- We provide round the clock customer support

- Convenient online submission

- Thorough peer review

- Inclusion in PubMed and all major indexing services

- Maximum visibility for your research

Submit your manuscript at www.biomedcentral.com/submit

) Biomed Central 